\title{
(อ) OPEN ACCESS \\ Clinical features to distinguish meningitis among young infants at a rural Kenyan hospital
}

\author{
Christina W Obiero (D) , 1,2 Neema Mturi, ${ }^{1}$ Salim Mwarumba, ${ }^{3}$ Moses Ngari, ${ }^{1,4}$ \\ Charles Newton, ${ }^{1,5}$ Michael Boele van Hensbroek, ${ }^{2}$ James Alexander Berkley
}

- Additional material is published online only. To view please visit the journal online (http://dx.doi.org/10.1136/ archdischild-2020-318913).

${ }^{1}$ Clinical Research Department, KEMRI-Wellcome Trust Research Programme, Kilifi, Kenya ${ }^{2}$ Department of Global Health, University of Amsterdam Faculty of Medicine, Amsterdam, NoordHolland, The Netherlands ${ }^{3}$ Department of Microbiology, KEMRI-Wellcome Trust Research Programme, Kilifi, Kenya ${ }^{4}$ The Childhood Acute Illness and Nutrition (CHAIN) Network, Nairobi, Kenya

${ }^{5}$ Department of Psychiatry, University of Oxford Centre for Tropical Medicine and Global Health, Oxford, UK

${ }^{6}$ Nuffield Department of Medicine, University of Oxford Centre for Tropical Medicine and Global Health, Oxford, UK

\section{Correspondence to}

Dr Christina W Obiero, Clinical Research Department, KEMRIWellcome Trust Research Programme, Kilifi 80108, Kenya; cobiero@kemri-wellcome.org

Received 24 January 2020

Revised 23 June 2020

Accepted 1 July 2020

Published Online First 20 August 2020

\section{Check for updates}

(c) Author(s) (or their employer(s)) 2021. Re-use permitted under CC BY. Published by BMJ.

To cite: Obiero CW, Mturi N, Mwarumba $\mathrm{S}$, et al.

Arch Dis Child

2021:106:130-136.

\begin{abstract}
Background Detection of meningitis is essential to optimise the duration and choice of antimicrobial agents to limit mortality and sequelae. In low and middleincome countries most health facilities lack laboratory capacity and rely on clinical features to empirically treat meningitis.
\end{abstract}

Objective We conducted a diagnostic validation study to investigate the performance of clinical features (fever, convulsions, irritability, bulging fontanel and temperature $\geq 39^{\circ} \mathrm{C}$ ) and WHO-recommended signs (drowsiness, lethargy, unconsciousness, convulsions, bulging fontanel, irritability or a high-pitched cry) in discriminating

meningitis in young infants.

Design Retrospective cohort study.

Setting Kilifi County Hospital.

Patients Infants aged $<60$ days hospitalised between 2012 and 2016.

Main outcome measure Definite meningitis defined as positive cerebrospinal fluid (CSF) culture, microscopy or antigen test, or leucocytes $\geq 0.05 \times 10 \wedge 9 / \mathrm{L}$.

Results Of 4809 infants aged $<60$ days included, 81 $(1.7 \%)$ had definite meningitis. WHO-recommended signs had sensitivity of $58 \%(95 \% \mathrm{Cl} 47 \%$ to $69 \%)$ and specificity of $57 \%(95 \% \mathrm{Cl} 56 \%$ to $59 \%)$ for definite meningitis. Addition of history of fever improved sensitivity to $89 \%$ ( $95 \% \mathrm{Cl} 80 \%$ to $95 \%$ ) but reduced specificity to $26 \%$ ( $95 \% \mathrm{Cl} 25 \%$ to $27 \%$ ). Presence of $\geq 1$ of 5 previously identified signs had sensitivity of $79 \%$ $(95 \% \mathrm{Cl} 69 \%$ to $87 \%)$ and specificity of $51 \%(95 \% \mathrm{Cl}$ $50 \%$ to $53 \%$ ).

Conclusions Despite a lower prevalence of definite meningitis, the performance of previously identified signs at admission in predicting meningitis was unchanged. Presence of history of fever improves the sensitivity of WHO-recommended signs but loses specificity. Careful evaluation, repeated assessment and capacity for lumbar puncture and CSF microscopy to exclude meningitis in most young infants with potential signs are essential to management in this age group.

\section{INTRODUCTION}

Meningitis is a life-threatening disease associated with significant mortality and disabling neuropsychological sequelae. ${ }^{1-4}$ Disease burden is highest in low and middle-income countries where about a quarter of children who survive vaccine-preventable meningitis develop postdischarge complications. ${ }^{56}$ Prompt recognition and treatment with appropriate antimicrobial coverage and cerebrospinal fluid (CSF) penetration for an adequate duration is critical to optimise outcomes.
What is already known on this topic?

- Meningitis is associated with significant mortality and long-term neurological impairment, particularly in low and middleincome countries where disease burden is highest and diagnostic resources constrained.

- We previously independently identified simple predictors of meningitis (fever, convulsions, irritability, bulging fontanel or temperature $\geq 39^{\circ} \mathrm{C}$ ) in young infants at our centre.

- Current management guidelines are based on limited evidence obtained prior to use of conjugate vaccines and may not be optimal given changes in disease epidemiology.

\section{What this study adds?}

- This study investigated the performance of previously identified clinical features and WHOrecommended signs in discriminating meningitis in young infants hospitalised at a rural hospital.

- Meningitis is less common than previously found but performance of clinical features in discriminating meningitis has not changed since the introduction of conjugate vaccines.

- Low specificity of clinical features means that the capacity for basic cerebrospinal fluid analysis is essential to avoid unnecessary treatment.

CSF culture is the gold standard diagnostic test for meningitis; however, it has limited sensitivity, is compromised by prior antibiotic exposure ${ }^{8}$ and is frequently unavailable or unreliable in resourcelimited hospitals. Changes in CSF cytological and biochemical parameters have diagnostic utility but rarely CSF may be normal in the presence of meningitis. ${ }^{910}$ Most health facilities in low and middleincome countries lack CSF diagnostic capacity and so management decisions are based on clinical presentation only.

Young infants typically present to hospital with subtle symptoms and signs, ${ }^{111}$ making it challenging for clinicians to decide when to perform a lumbar puncture (LP) (if laboratory facilities exist) or continue empirical antibiotics. The WHO advises suspecting meningitis if an infant: (1) is drowsy, lethargic or unconscious; (2) has convulsions; (3) has a bulging fontanel; (4) is irritable, or (5) has a high-pitched cry. ${ }^{12}$ These guidelines are based on 
limited evidence collected prior to widespread use of conjugate vaccines, ${ }^{13}$ including a study that reported clinical signs not specific to meningitis. ${ }^{12}$

Between 2001 and 2007, we conducted a study of clinical features associated with meningitis among infants aged $<60$ days at Kilifi County Hospital $(\mathrm{KCH})$ and found history of fever, convulsions, irritability, bulging fontanel or temperature $\geq 39^{\circ} \mathrm{C}$ to be useful indicators of meningitis. ${ }^{14}$ These results, including the WHO recommendation, were incorporated into Kenyan national guidelines. ${ }^{15}$ However, since the introduction of conjugate Haemophilus influenzae type b (Hib) vaccine in 2001, an $89 \%$ reduction in Hib meningitis in Kenyan children has been observed. ${ }^{16}$ Similarly, 10 -valent pneumococcal conjugate vaccine (PCV-10) was introduced in 2011 and was associated with significant reduction in nasopharyngeal carriage of vaccine serotypes, ${ }^{17}$ and incidence and mortality from pneumococcal meningitis. ${ }^{18-20}$ Additionally, the introduction of a voucher scheme and free maternity care in 2013 in Kenya has resulted in a greatly increased number of hospital deliveries and admissions directly to paediatric care. ${ }^{21}$ Although intrapartum antibiotic prophylaxis is recommended in the presence of risk factors for infection such as prolonged rapture of membranes $\geq 18$ hours, maternal screening for group B streptococci (GBS) is not included in the Kenya national guidelines, ${ }^{22}$ despite high incidence of early-onset neonatal infection secondary to $\mathrm{GBS}^{23}$

Thus, changes in both epidemiology and patient profile may have altered the associations between clinical features and meningitis, hence clinical decision rules derived from earlier studies may no longer be optimal. We therefore performed a revalidation study of clinical features at admission to hospital in infants aged $<60$ days, examining those identified in the previous study at our centre and those recommended by the WHO.

\section{METHODS}

Location and participants

$\mathrm{KCH}$ is a government hospital located on the Kenyan coast serving a mostly rural population. Routine vaccination with $\mathrm{Hib}$ and PCV-10 vaccines are provided free of charge at government health facilities as a three-dose primary series without a booster dose at 6,10 and 14 weeks of age. All infants $<60$ days old hospitalised at $\mathrm{KCH}$ between 1 January 2012 and 31 December 2016 were included in this retrospective cohort study.

\section{Procedures}

All infants were systematically assessed by trained clinicians at admission and standardised demographic and clinical data were prospectively collected and entered on a surveillance database in real time. Laboratory investigations done at admission on all infants included haemogram, blood slide for malaria parasites and blood culture. Infants presenting with signs suggestive of meningitis underwent LP and were started on broad-spectrum antibiotics according to $\mathrm{WHO}^{24}$ and Kenyan national guidelines. ${ }^{15}$ LP was deferred in infants with cardiorespiratory compromise or signs of raised intracranial pressure. ${ }^{25}$ Infants were assessed daily by clinicians and an LP performed once stable if LP had been delayed at admission, or if an infant developed new clinical features suggestive of meningitis during hospitalisation.

\section{Laboratory analysis}

CSF examination included leucocyte count, Gram and/or Indian ink staining and latex antigen agglutination tests (Wellcogen Bacterial Antigen kit for Streptococcus pneumoniae, H. influenzae, Neisseria meningitidis and CrAg Lateral Flow Assay kit Ref CR2003 for Cryptococcus neoformans). All CSF and blood samples were cultured as previously described and pathogens identified using standard methods, including antimicrobial susceptibility testing. ${ }^{14} 26$ Known commensals including coagulase-negative staphylococci were considered non-significant. CSF protein and glucose and concurrent blood glucose were measured on an Instrument Laboratory Aries analyser (Werfen, Germany).

Sample processing and analysis was performed at the KEMRI Centre for Geographic Medicine (Coast) laboratory which is externally monitored for quality assurance by the UK External Quality Assessment Service and accredited in Good Clinical Laboratory Practice by Qualogy, UK.

\section{Definitions}

For this analysis, we defined definite meningitis according to the criteria used in our previous study ${ }^{14}$ : (1) positive CSF culture for a known pathogen; or (2) organisms observed on CSF Gram stain microscopy; or (3) positive CSF antigen test; or (4) CSF leucocytes $\geq 0.05 \times 10 \wedge 9 / \mathrm{L} 50$ cells/ $\mu$ L. Possible meningitis was defined in infants without definite meningitis as: CSF leucocytes $\geq 0.02 \times 10 \wedge 9 / \mathrm{L}$ in infants aged $0-28$ days, and CSF leucocytes $\geq 0.01 \times 10 \wedge 9 / \mathrm{L}$ in infants aged $28-59$ days. Infants not meeting either criteria were defined as no meningitis. Possible meningitis and the narrow microbiological criteria for definite meningitis (positive CSF culture, antigen test or microscopy, or CSF leucocytes $\geq 0.05 \times 10 \wedge 9 / \mathrm{L}$ plus positive blood culture) were used for sensitivity analysis.

\section{Statistical analysis}

We extracted data from the surveillance database. Infants who died before an LP had been performed were then excluded as we could not ascertain their meningitis status. We analysed data from all infants and then separately examined those 0-6 days and 7-59 days because of potential differences in aetiology and clinical presentation. ${ }^{14}$

We calculated the prevalence of meningitis and tabulated the frequency distribution of CSF findings, including pathogens identified and the highest CSF criterion for definite meningitis attained in the order of the four criteria given above.

We examined the performance of the previously identified clinical features (history of fever, convulsions, irritability, bulging fontanel and temperature $\left.\geq 39^{\circ} \mathrm{C}\right)^{14}$ and the WHOrecommended signs ${ }^{24}$ by calculating their sensitivity, specificity, positive predictive value (PPV), negative predictive value (NPV) and area under receiver operating characteristic (ROC) curve for definite meningitis versus no meningitis.

We calculated the number of LPs needed to identify one case of definite meningitis using each combination of features as the inverse of the risk difference obtained by subtracting the risk of meningitis in the group with indicator(s) of interest from risk of meningitis in the group without indicator(s) of interest. As a sensitivity analysis we then repeated these analyses for possible or definite meningitis versus no meningitis.

Proportions were compared using the $\chi^{2}$ test or Fisher's exact test, while continuous variables were compared using 


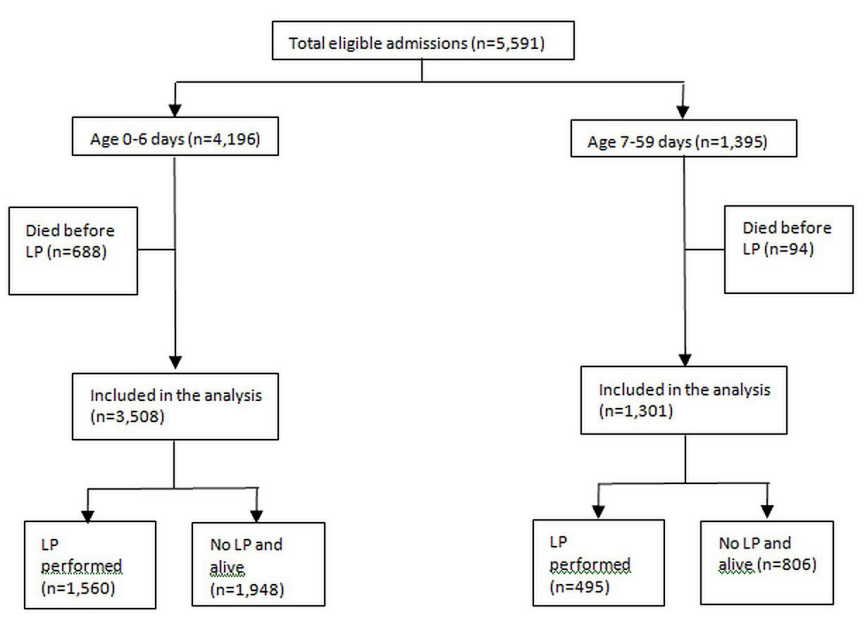

Figure 1 Flow chart of study participants. LP, lumbar puncture.

Wilcoxon rank-sum test. We performed analyses using Stata V.15 (StataCorp, USA).

\section{RESULTS}

During the study period, 5591 infants were admitted, of which 4196 (75\%) were aged 0-6 days. Three thousand two hundred and fifty-three (58\%) infants were born at $\mathrm{KCH}$, including 2747 (65\%) of those aged 0-6 days of whom 2476 (90\%) were hospitalised within the first 72 hours of life. Overall, 853/5591 (15\%) infants died during hospitalisation and $640 / 853(75 \%)$ deaths occurred during the first day of life. Seven hundred and eighty-two (92\%) of 853 deaths occurred before an LP had been performed and were excluded from this analysis (figure 1). Thus, 4809 infants of which 3508 (73\%) were aged 0-6 days were included in the analysis.

Eighty-one (1.7\%) infants had definite meningitis; 39/3508 (1.1\%) aged 0-6 days and 42/1301 (3.2\%) aged 7-59 days $(\mathrm{p}<0.001)$. Eighteen $(22 \%)$ infants had positive CSF culture (table 1) of which eight had a positive blood culture and six grew similar organisms (four GBS, one group A streptococcus and one Escherichia coli) in blood and CSF. GBS was the most common CSF isolate followed by E. coli and Klebsiella pneumoniae. Two infants had Hib and one had pneumococcal meningitis; none had positive CSF or blood cultures. Twentyseven $(33 \%)$ of the 81 definite meningitis cases had visibly turbid CSF. Three of 11 infants with positive blood culture had CSF leucocytes $\geq 0.05 \times 10 \wedge 9 / \mathrm{L}$. Seven $(8.6 \%)$ infants with definite meningitis died during admission compared with $64(1.4 \%)$ in the non-meningitis group, $\mathrm{p}<0.001$. Online supplementary table S1 shows the meningitis cases and the number of LPs during our study period versus our previous analysis.

\section{Clinical features at admission}

History of fever, bulging fontanel, axillary temperature $\geq 39^{\circ} \mathrm{C}$ and irritability were associated with definite meningitis among infants aged 0-6 days (table 2). Bulging fontanel, convulsions and irritability were associated with definite

Table 1 Diagnostic criteria for meningitis and bacterial species detected

\begin{tabular}{|c|c|c|c|c|}
\hline Diagnostic criteria & Ages $0-6$ days $(3508 / 4809,73 \%)$ & Ages $7-59$ days (1301/4809, 27\%) & Total positives & Highest criteria for meningitis \\
\hline \multicolumn{5}{|l|}{ CSF culture } \\
\hline \multicolumn{5}{|l|}{ Gram positive } \\
\hline Group B streptococci & 0 & 7 & $7^{*}$ & 7 \\
\hline Group A streptococci & 0 & 1 & $1 \dagger$ & 1 \\
\hline \multicolumn{5}{|l|}{ Gram negative } \\
\hline Escherichia coli & 2 & 3 & $5 \ddagger$ & 5 \\
\hline Klebsiella pneumonia & 1 & 1 & $2 \S$ & 2 \\
\hline Klebsiella oxytoca & 0 & 1 & 1 & 1 \\
\hline Enterobacter aerogenes & 0 & 1 & 1 & 1 \\
\hline Citrobacter sp & 1 & 0 & 19 & 1 \\
\hline Total & 4 & 14 & 18 & 18 \\
\hline \multicolumn{5}{|l|}{ Latex antigen test } \\
\hline Streptococcus pneumoniae & 0 & 1 & $1^{* *}$ & 1 \\
\hline Haemophilus influenzae & 1 & 1 & $2+\dagger$ & 2 \\
\hline Cryptococcus neoformans & 1 & 0 & 1 & 1 \\
\hline Total positive antigen test & 2 & 2 & 4 & 4 \\
\hline \multicolumn{5}{|l|}{ Gram stain } \\
\hline Gram-positive cocci & 0 & 6 & 6 & 0 \\
\hline Gram-negative rods & 5 & 1 & 6 & 2 \\
\hline Total & 5 & 7 & $12 \ddagger \ddagger$ & 2 \\
\hline Indian ink & 1 & 0 & 1 & 1 \\
\hline CSF WCC $\geq 0.05 \times 10 \wedge 9 / L$ & 33 & 37 & 70 & 56 \\
\hline Total & & & & 81 \\
\hline
\end{tabular}

*6/7 had WCC $\geq 0.05 \times 10 \wedge 9 / \mathrm{L}$ and 5/7 had positive Gram stain.

HHad WCC $\geq 0.05 \times 10 \wedge 9 / \mathrm{L}$ and positive Gram stain.

$\$ 1 / 5$ had Streptococcus sp isolated as well; $3 / 5$ had WCC $\geq 0.05 \times 10 \wedge 9 / L ; 2 / 5$ had positive Gram stain.

$\S 1 / 2$ had WCC $\geq 0.05 \times 10 \wedge 9 / L ;$ one had positive Gram stain.

१Had WCC $\geq 0.05 \times 10 \wedge 9 / \mathrm{L}$ and positive Gram stain.

${ }^{* *} \mathrm{Had}$ WCC $\geq 0.05 \times 10 \wedge 9 / \mathrm{L}$.

t+1/2 had WCC $>0.05 \times 10 \wedge 9 / \mathrm{L}$.

$\ddagger \ddagger 10 / 12$ had positive CSF culture; $9 / 10$ had WCC $\geq 0.05 \times 10 \wedge 9 / \mathrm{L}$.

CSF, cerebrospinal fluid; WCC, white cell count. 
Table 2 Clinical history and examination findings among neonates and young infants

\begin{tabular}{|c|c|c|c|c|c|c|}
\hline \multirow[b]{2}{*}{ Characteristic } & \multicolumn{3}{|c|}{ Ages $0-6$ days } & \multicolumn{3}{|c|}{ Ages $7-59$ days } \\
\hline & No meningitis $(n=3469)$ & Meningitis $(n=39)$ & $P$ value* & No meningitis $(n=1259)$ & Meningitis $(n=42)$ & $P$ value* \\
\hline \multicolumn{7}{|c|}{ Bulging fontanel } \\
\hline No & 3419 (99) & $37(95)$ & $<0.001$ & $1233(98)$ & $33(79)$ & $<0.001$ \\
\hline Yes & $15(0.4)$ & $2(5.1)$ & & $11(0.9)$ & $9(21)$ & \\
\hline Missing & $35(1.0)$ & $0(0)$ & & $15(1.2)$ & $0(0)$ & \\
\hline \multicolumn{7}{|l|}{ Convulsions } \\
\hline No & 3269 (94) & $34(87)$ & 0.063 & $1162(92)$ & $31(74)$ & $<0.001$ \\
\hline Yes & $168(4.8)$ & $5(13)$ & & $82(6.5)$ & $11(26)$ & \\
\hline Missing & $32(0.9)$ & $0(0)$ & & $15(1.2)$ & $0(0)$ & \\
\hline \multicolumn{7}{|c|}{ Axillary temperature $\left({ }^{\circ} \mathrm{C}\right)$} \\
\hline$<36$ & $1061(31)$ & $4(10)$ & 0.021 & $77(6.1)$ & $1(2.4)$ & 0.269 \\
\hline $36-38.9$ & $2202(63)$ & $30(77)$ & & $1092(87)$ & $35(83)$ & \\
\hline$\geq 39$ & $199(5.7)$ & $5(13)$ & & $89(7.1)$ & $6(14)$ & \\
\hline Missing & $7(0.2)$ & $0(0)$ & & $1(0.1)$ & $0(0)$ & \\
\hline \multicolumn{7}{|c|}{ Agitation/irritability } \\
\hline No & $3375(97)$ & $33(85)$ & $<0.001$ & $1198(95)$ & $36(86)$ & 0.002 \\
\hline Yes & $59(1.7)$ & $6(15)$ & & $46(3.7)$ & $6(14)$ & \\
\hline Missing & $35(1.0)$ & $0(0)$ & & $15(1.2)$ & $0(0)$ & \\
\hline \multicolumn{7}{|c|}{ History of fevert } \\
\hline No & $2086(60)$ & $14(36)$ & 0.006 & $470(37)$ & $9(21)$ & 0.074 \\
\hline Yes & $1350(39)$ & $25(64)$ & & $774(61)$ & $33(79)$ & \\
\hline Missing & $33(1.0)$ & $0(0)$ & & $15(1.1)$ & $0(0)$ & \\
\hline \multicolumn{7}{|c|}{ Drowsy, lethargic or unconscious } \\
\hline No & $2626(76)$ & 27 (69) & 0.466 & $1048(83)$ & 31 (74) & 0.148 \\
\hline Yes & $808(23)$ & $12(31)$ & & $196(16)$ & $11(26)$ & \\
\hline Missing & $35(1.0)$ & $0(0)$ & & $15(1.2)$ & $0(0)$ & \\
\hline \multicolumn{7}{|l|}{ Abnormal cry } \\
\hline No & $2489(72)$ & $33(85)$ & 0.093 & 846 (67) & $31(74)$ & 0.587 \\
\hline Yes & $945(27)$ & $5(13)$ & & $118(9.4)$ & $4(9.5)$ & \\
\hline Missing & $35(1.0)$ & $1(2.6)$ & & $295(23)$ & $7(! 7)$ & \\
\hline
\end{tabular}

Data are $n(\%)$.

Group percentages may not add to $100 \%$ due to rounding off.

*Univariate comparison of characteristics.

tElevated tactile temperature as reported by the parent or guardian.

meningitis in older infants. A bulging fontanel, stiff neck and inability to breast feed were each observed in only $5 \%$ of meningitis cases.

\section{Performance of clinical features in all infants}

Previously identified signs

Sixty-four (2.7\%) of 2377 infants presenting with one or more of history of fever, irritability, axillary temperature $\geq 39^{\circ} \mathrm{C}$, convulsions or bulging fontanel had definite meningitis compared with $17 / 2432(0.7 \%)$ infants lacking these features $(\mathrm{p}<0.001)$ : sensitivity $79 \%(95 \%$ CI $69 \%$ to $87 \%)$, specificity 51\% (95\% CI 50\% to 53\%), PPV 2.7\% (95\% CI $2.1 \%$ to $3.4 \%$ ), NPV $99 \%$ (95\% CI $99 \%$ to $100 \%)$. Fifty infants (95\% CI 37 to 79 ) presenting with one or more of these clinical features would need to undergo an LP for each case of meningitis to be identified (table 3 ).

WHO-recommended signs or a history of fever

Forty-seven $(2.3 \%)$ of 2072 infants presenting with one or more of WHO-recommended signs had definite meningitis compared with $34 / 2737(1 \%)$ infants lacking these signs $(\mathrm{p}=0.006)$ : sensitivity $58 \%$ (95\% CI $47 \%$ to $69 \%$ ), specificity $57 \%$ (95\% CI $56 \%$ to $59 \%$ ), PPV $2.3 \%$ (95\% CI $1.7 \%$ to $3 \%$ ) and NPV $99 \%$ (95\% CI $98 \%$ to $99 \%$ ). Ninety-eight infants (95\% CI 56 to
381) presenting with one or more of WHO-recommended signs would need to undergo an LP for each meningitis case to be identified (table 3). Addition of history of fever to these WHO signs resulted in sensitivity $89 \%$ (95\% CI $80 \%$ to $95 \%$ ), specificity $26 \%$ (95\% CI $25 \%$ to $27 \%$ ), PPV $2 \%$ (95\% CI $1.6 \%$ to $2.5 \%)$ and NPV 99\% (95\% CI 99\% to 100\%).

Clinical features in infants 0-6 days old compared with infants 7-59 days old

Previously identified signs were less sensitive but more specific in detecting meningitis in infants in the first week of life than among infants 7-59 days old (online supplementary tables S2 and S3). WHO-recommended signs had similar sensitivity and specificity in both age groups. History of fever markedly improved the sensitivity of WHO-recommended signs but resulted in low specificity in both age groups.

The overall area under the ROC curve for previously identified signs was 0.72 (95\% CI 0.66 to 0.78$)$ and there was no evidence that this differed between infants aged 0-6 days and aged 7-59 days $(p=0.19)$ (figure 2$)$.

\section{Sensitivity analysis}

One hundred and twenty-six infants had possible or definite meningitis. Fewer LPs were needed to identify a single case of 
Table 3 Performance of indicators of meningitis among all infants $0-59$ days old

\begin{tabular}{|c|c|c|c|c|c|c|c|}
\hline Indicators & $\begin{array}{l}\text { Number } \\
\text { with } \\
\text { indicator }\end{array}$ & $\begin{array}{l}\text { Number with } \\
\text { meningitis }\end{array}$ & Sensitivity $(95 \% \mathrm{Cl})$ & Specificity (95\% Cl) & PPV $(95 \% \mathrm{Cl})$ & $\operatorname{NPV}(95 \% \mathrm{Cl})$ & NN LP $(95 \%$ Cl) \\
\hline \multicolumn{8}{|l|}{ Previously identified } \\
\hline Bulging fontanel & 37 & 11 & 13.6 (7.0 to 23.0$)$ & 99.4 (99.2 to 99.6$)$ & 29.7 (15.9 to 47.0$)$ & 98.5 (98.1 to 98.8 ) & 4 (2 to 7$)$ \\
\hline Convulsions or any of the above & 292 & 24 & 29.6 (20.0 to 40.8$)$ & 94.3 (93.6 to 95.0$)$ & $8.2(5.3$ to 12.0$)$ & 98.7 (98.4 to 99.0$)$ & 14 (10 to 26$)$ \\
\hline $\begin{array}{l}\text { Axillary temperature } \geq 39^{\circ} \mathrm{C} \text { or } \\
\text { any of the above }\end{array}$ & 555 & 32 & $39.5(28.8$ to 51.0$)$ & 88.9 (88.0 to 89.8 ) & $5.8(4.0$ to 8.0$)$ & 98.8 (98.5 to 99.1 ) & 22 (15 to 38$)$ \\
\hline $\begin{array}{l}\text { Agitation/irritability or any of } \\
\text { the above }\end{array}$ & 640 & 37 & 45.7 (34.6 to 57.1$)$ & 87.2 (86.3 to 88.2 ) & 5.8 (4.1 to 7.9$)$ & 98.9 (98.6 to 99.2$)$ & 21 (15 to 35$)$ \\
\hline $\begin{array}{l}\text { History of fever or any of the } \\
\text { above }\end{array}$ & 2377 & 64 & $79.0(68.5$ to 87.3$)$ & 51.1 (49.6 to 52.5 ) & 2.7 (2.1 to 3.4$)$ & 99.3 (98.9 to 99.6$)$ & 50 (37 to 79 ) \\
\hline \multicolumn{8}{|l|}{ WHO recommended } \\
\hline $\begin{array}{l}\text { One or more of the WHO- } \\
\text { suggested signs }\end{array}$ & 2072 & 47 & 58.0 (46.5 to 68.9$)$ & 57.2 (55.7 to 58.6$)$ & 2.3 (1.7 to 3.0$)$ & 98.8 (98.3 to 99.1$)$ & 98 (56 to 381$)$ \\
\hline $\begin{array}{l}\text { One or more of the WHO- } \\
\text { suggested signs or history of } \\
\text { fever }\end{array}$ & 3566 & 72 & $88.9(80.0$ to 94.8$)$ & 26.1 (24.9 to 27.4 ) & 2.0 (1.6 to 2.5$)$ & 99.3 (98.6 to 99.7 ) & 77 (51 to 157$)$ \\
\hline
\end{tabular}

NN LP, number needed to lumbar puncture to identify one case of definite meningitis; NPV, negative predictive value; PPV, positive predictive value.

possible or definite meningitis compared with a cut-off $\geq 50$ / $\mu \mathrm{L}$ in all infants (online supplementary table S4). The overall area under the ROC curve for previously identified signs was 0.69 (95\% CI 0.64 to 0.74 ) and there was no evidence that this differed between infants aged 0-6 days and aged 7-59 days $(\mathrm{p}=0.17)$.

For the $28(0.6 \%)$ infants with definite meningitis based on microbiological criteria, point estimates for sensitivity and specificity for previously identified signs, and WHO-recommended signs or a history of fever respectively were similar to the main analysis and more LPs were needed to identify a single case of meningitis (online supplementary table S5).

\section{DISCUSSION}

This study aimed to determine if clinical features at admission to hospital that were found to discriminate young infants with meningitis before widespread use of conjugate vaccines in low

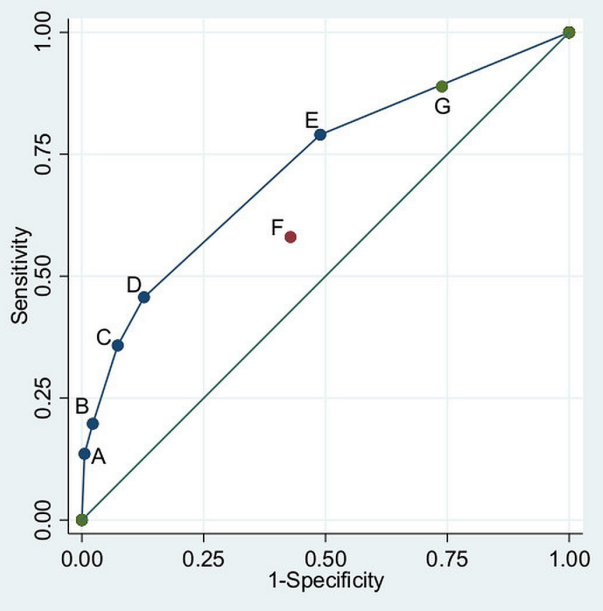

Figure 2 Area under receiver operating characteristic (ROC) curve for previously identified signs and WHO-recommended signs of meningitis or history of fever in infants aged $0-59$ days. (A) Bulging fontanel. (B) Convulsions. (C) Axillary temperature $\geq 39^{\circ} \mathrm{C}$. (D) Agitation/irritability. (E) History of fever. (F) WHO-recommended signs. (G) WHO-recommended signs or history of fever. and middle-income countries are still applicable for decisionmaking. Overall, 1.7\% infants included in our study had definite meningitis, lower than $4.2 \%$ in $2006-2007$ and $4.1 \%$ in 2001 2005 previously at our centre using the same definition and inclusion criteria. ${ }^{14}$ The number of infants hospitalised at $\mathrm{KCH}$ has increased, predominantly due to increased admissions on the first day of life related to uptake of free maternity care. ${ }^{14} 21$ Meningitis cases decreased with time despite an increase in the number of LPs performed. Comparing the present study (20122016) to our previous study (1994-1998), ${ }^{27}$ there were 1 vs 24 cases of $S$. pneumoniae and 2 vs 11 cases of $H$. influenzae meningitis demonstrating an effect of herd immunity from conjugate vaccination on case load.

In addition to limited CSF diagnostic capacity, ancillary tests that may be helpful in stratifying infants at risk of meningitis such as peripheral blood leucopenia, ${ }^{28}$ absolute neutrophilia ${ }^{29}$ and biomarkers (eg, procalcitonin) $)^{30}$ are usually unavailable and have not been validated in our setting. This further underscores the need to optimise clinical guidelines to identify infants needing urgent treatment.

While the presence of one or more of the previously identified signs missed less cases than WHO-recommended signs alone ( $21 \%$ vs $42 \%)$, history of fever improved the performance of WHO signs. Our results were similar to those reported by our previous study, ${ }^{14}$ suggesting that although meningitis is now less common, performance of these signs, including those recommended by $\mathrm{WHO}$, has not significantly changed over time. Of importance, none of these signs were highly specific in discriminating meningitis at admission, hence careful clinical review and LP are essential, especially in consideration of duration of antibiotics once started.

Capacity for CSF analysis is often unavailable in resourcelimited settings ${ }^{31}$ and more LPs are now needed to identify a single meningitis case than previously, especially with the narrowest meningitis case definition. ${ }^{14}$ There are limited data on the 'acceptable' number of LPs needed to diagnose a single case of meningitis in young infants, with most studies focusing on the clinical utility of LPs in older children with seizures. ${ }^{32}$

Typically, about 50\% meningitis deaths occur within the first 24 hours of admission and postmortem LP may be useful for surveillance and studies of aetiology, ${ }^{27}$ but undertaking LP 
promptly is vital for diagnosis. The low specificity of clinical signs leads to overdiagnosis. However, given the high risk of morbidity and mortality associated with bacterial meningitis and the contribution to development of antimicrobial resistance of overtreatment, any reluctance to perform LPs, even where full laboratory support is unavailable, needs to be addressed as failing to do so will miss $10 \%-40 \%$ of cases and/or overtreat the vast majority of infants with indicator signs (online supplementary table S4). Meningitis is now less common, and more difficult to exclude purely on clinical grounds and clinicians should maintain a low threshold for doing LPs, such as in all infants with fever in addition to more specific signs, especially in $0-6$ day-olds.

Thirty seven per cent of meningitis cases in our study had turbid CSF, similar to $28 \%$ cases in 2001-2005 ( $\mathrm{p}=0.18$ ). CSF leucocyte count may fail to discriminate infants with cultureproven meningitis from those without,${ }^{10}$ but together with visual turbidity would have identified $71(88 \%)$ of definite cases and 118 (94\%) of possible cases. Sensitivity analysis done in our study with a lower cut-off $(\geq 20 / \mu \mathrm{L})$, which has been shown to provide sufficient diagnostic precision for culture-proven meningitis, ${ }^{7}$ did not alter our results. Support to establish basic CSF cell counting and Gram stain in resource-limited settings to optimise antimicrobial treatment is essential to providing inpatient paediatric services.

\section{Limitations}

We lacked data on prehospital antibiotic exposure which has been shown to lower CSF culture yield. ${ }^{83}$ Potential misclassification of infants with negative CSF findings or asymptomatic infants may have diminished the validity of clinical features studied. Infants who died prior to an LP were excluded from this analysis, however, we did not aim to estimate the overall burden of meningitis, rather to address the challenges faced in clinical practice among infants in whom a decision to admit to hospital had been made. Delays in presentation to hospital ${ }^{34}$ and rapid disease progression may have led to early mortality before LP. This may have a pathogen-specific impact on our findings as, for example, GBS mortality commonly occurs in the first few hours of life. ${ }^{23}$

\section{CONCLUSIONS}

Meningitis is an uncommon but important diagnosis in young infants. Despite declining incidence, clinical features of meningitis do not perform less well now than in the preconjugate vaccine era. However, clinicians and policymakers should be aware of the number of LPs or empirical treatments needed for each case of definite meningitis identified. The clinical signs currently recommended by WHO to guide decisions to perform an LP and initiate antibiotics poorly discriminate infants with meningitis, particularly in neonates aged $<1$ week. History of fever is an important indicator and clinicians should not rely on 'classical' signs such as neck stiffness or bulging fontanel only. Even the best-performing clinical decision rule fails to identify all cases when applied at admission and has poor specificity, hence it is important that all young infants hospitalised with serious illness undergo an LP.

Acknowledgements This study is published with the permission of the Director of Kenya Medical Research Institute. Surveillance at KCH was undertaken at the KWTRP by members of the KWTRP medical, nursing, laboratory and computing team who participated in patient care, data collection and data storage. We thank all KWTRP staff and KCH patients whose data were included in this analysis.
Contributors CWO, MN, CN, MBvH and JAB contributed to the conception and design of the study. CWO, NM and JAB contributed to inpatient care and data collection. SM was responsible for laboratory analysis. CWO, NM, SM, MN, CN $\mathrm{MBvH}$ and $\mathrm{JAB}$ contributed to the analysis and interpretation of data. $\mathrm{CWO}, \mathrm{MBVH}$ and $J A B$ contributed to the drafting of the article. All authors read and approved the final manuscript.

Funding This work was supported by the Wellcome Trust, UK core grant to KEMRIWellcome Trust Research Programme (grant 203077/Z/16/Z). CWO is supported by the Drugs for Neglected Diseases initiative (grant OXF-DND02). JAB is supported by the Bill \& Melinda Gates Foundation within the Childhood Acute IIIness and Nutrition (CHAIN) Network (grant OPP1131320) and by the MRC/DFID/Wellcome Trust Joint Global Health Trials scheme (grant MR/M007367/1)

Disclaimer The views expressed in this manuscript are those of the authors and not necessarily those of the KEMRI, or the Wellcome Trust.

\section{Competing interests None declared.}

\section{Patient consent for publication Not required.}

Ethics approval Collection of surveillance data included in this analysis was reviewed and approved by the Kenya Medical Research Institute Scientific Steering Committee (KEMRI SSC 1433). This retrospective analysis was reviewed and approved by the KEMRI SSC (KEMRI SSC 3001).

Provenance and peer review Not commissioned; externally peer reviewed.

Data availability statement Data are available upon reasonable request. The data set used and analysed during the current study is available from the KEMRIWellcome Trust Research Programme (KWTRP) Data Governance Committee (DGC) on reasonable request (dgc@kemri-wellcome.org), ensuring protection of the privacy, rights and interests of research participants and primary researchers, and upholding transparency and accountability. KWTRP is the custodian of the data used in this analysis and the KWTRP DGC oversees the internal data repository.

Open access This is an open access article distributed in accordance with the Creative Commons Attribution 4.0 Unported (CC BY 4.0) license, which permits others to copy, redistribute, remix, transform and build upon this work for any purpose, provided the original work is properly cited, a link to the licence is given, and indication of whether changes were made. See: https://creativecommons.org/ licenses/by/4.0/.

\section{ORCID iDs}

Christina W Obiero http://orcid.org/0000-0002-9321-0183 James Alexander Berkley http://orcid.org/0000-0002-1236-849X

\section{REFERENCES}

1 Mann K, Jackson MA. Meningitis. Pediatr Rev 2008;29:417-30.

2 Khowaja AR, Mohiuddin S, Cohen AL, et al. Mortality and neurodevelopmental outcomes of acute bacterial meningitis in children aged $<5$ years in Pakistan. J Pediat 2013;163:S86-91.

3 Christie D, Rashid $\mathrm{H}$, El-Bashir $\mathrm{H}$, et al. Impact of meningitis on intelligence and development: a systematic review and meta-analysis. PLoS One 2017;12:e0175024.

4 Hudson LD, Viner RM, Christie D. Long-term sequelae of childhood bacterial meningitis. Curr Infect Dis Rep 2013;15:236-41.

5 Ramakrishnan M, Ulland AJ, Steinhardt LC, et al. Sequelae due to bacterial meningitis among African children: a systematic literature review. BMC Med 2009;7:47.

6 Edmond K, Clark A, Korczak VS, et al. Global and regional risk of disabling sequelae from bacterial meningitis: a systematic review and meta-analysis. Lancet Infect Dis 2010;10:317-28

7 Manning L, Laman M, Mare T, et al. Accuracy of cerebrospinal leucocyte count, protein and culture for the diagnosis of acute bacterial meningitis: a comparative study using Bayesian latent class analysis. Trop Med Int Health 2014;19:1520-4.

8 Kanegaye JT, Soliemanzadeh P, Bradley JS. Lumbar puncture in pediatric bacterial meningitis: defining the time interval for recovery of cerebrospinal fluid pathogens after parenteral antibiotic pretreatment. Pediatrics 2001;108:1169-74.

9 Polk DB, Steele RW. Bacterial meningitis presenting with normal cerebrospinal fluid. Pediatr Infect Dis J 1987:6:1040-2.

10 Garges HP, Moody MA, Cotten CM, et al. Neonatal meningitis: what is the correlation among cerebrospinal fluid cultures, blood cultures, and cerebrospinal fluid parameters? Pediatrics 2006;117:1094-100

$11 \mathrm{Ku} \mathrm{LC}$, Boggess KA, Cohen-Wolkowiez M. Bacterial meningitis in infants. Clin Perinatol 2015;42:vii-viii:29-45.

12 Young Infants Clinical Signs Study Group. Clinical signs that predict severe illness in children under age 2 months: a multicentre study. Lancet 2008;371:135-42.

13 Clinical prediction of serious bacterial infections in young infants in developing countries. The who young infants Study Group. Pediatr Infect Dis J 1999;18:S23-31.

14 Mwaniki MK, Talbert AW, Njuguna P, et al. Clinical indicators of bacterial meningitis among neonates and young infants in rural Kenya. BMC Infect Dis 2011;11:301.

$15 \mathrm{MOH}$. Basic paediatric protocols for ages up to 5 years. 4 edn, 2016. 
16 Cowgill KD, Ndiritu M, Nyiro J, et al. Effectiveness of Haemophilus influenzae type B conjugate vaccine introduction into routine childhood immunization in Kenya. JAMA 2006;296:671-8.

17 Hammitt LL, Akech DO, Morpeth SC, et al. Population effect of 10-valent pneumococcal conjugate vaccine on nasopharyngeal carriage of Streptococcus pneumoniae and non-typeable Haemophilus influenzae in Kilifi, Kenya: findings from cross-sectional carriage studies. Lancet Glob Health 2014;2:e397-405.

18 Gessner BD, Adegbola RA. The impact of vaccines on pneumonia: key lessons from Haemophilus influenzae type B conjugate vaccines. Vaccine 2008;26 Suppl 2:B3-8.

19 GBD 2015 Child Mortality Collaborators. Global, regional, national, and selected subnational levels of stillbirths, neonatal, infant, and under-5 mortality, 19802015: a systematic analysis for the global burden of disease study 2015. Lancet 2016;388:1725-74.

20 Wahl B, O'Brien KL, Greenbaum A, et al. Burden of Streptococcus pneumoniae and Haemophilus influenzae type $B$ disease in children in the era of conjugate vaccines: global, regional, and national estimates for 2000-15. Lancet Glob Health 2018:6:e744-57.

21 Wamalwa EW. Implementation challenges of free maternity services policy in Kenya: the health workers' perspective. Pan Afr Med J 2015;22:375.

22 Okola VO. Effectiveness of a group B streptococcus (GBS) protocol on GBS screening and intrapartum antibiotic prophylaxis at Kenyatta national Hospital. University of Nairobi, 2017.

23 Seale AC, Koech AC, Sheppard AE, et al. Maternal colonization with Streptococcus agalactiae and associated stillbirth and neonatal disease in coastal Kenya. Nat Microbiol 2016;1:16067.

24 WHO. Pocket book of hospital care for children: guidelines for the management of common childhood illnesses. 2 edn, 2013.
25 Srinivasan L, Harris MC, Shah SS. Lumbar puncture in the neonate: challenges in decision making and interpretation. Semin Perinatol 2012;36:445-53.

26 Berkley JA, Mwangi I, Mellington F, et al. Cerebral malaria versus bacterial meningitis in children with impaired consciousness. QJM 1999;92:151-7.

27 Mwangi I, Berkley J, Lowe B, et al. Acute bacterial meningitis in children admitted to a rural Kenyan Hospital: increasing antibiotic resistance and outcome. Pediatr Infect Dis J 2002;21:1042-8.

28 Bonsu BK, Harper MB. A low peripheral blood white blood cell count in infants younger than 90 days increases the odds of acute bacterial meningitis relative to bacteremia. Acad Emerg Med 2004;11:1297-301.

29 Nigrovic LE, Malley R, Kuppermann N. Meta-analysis of bacterial meningitis score validation studies. Arch Dis Child 2012;97:799-805.

30 Kuppermann N, Dayan PS, Levine DA, et al. A clinical prediction rule to identify febrile infants 60 days and younger at low risk for serious bacterial infections. JAMA Pediatr 2019;173:342-51

31 English M, Gathara D, Mwinga S, et al. Adoption of recommended practices and basic technologies in a low-income setting. Arch Dis Child 2014;99:452-6.

32 Najaf-Zadeh A, Dubos F, Hue V, et al. Risk of bacterial meningitis in young children with a first seizure in the context of fever: a systematic review and meta-analysis. PLoS One 2013;8:e55270.

33 Sáez-Llorens X, McCracken GH. Bacterial meningitis in children. Lancet 2003:361:2139-48.

34 Molyneux E, Walsh A, Phiri A, et al. Acute bacterial meningitis in children admitted to the Queen Elizabeth central Hospital, Blantyre, Malawi in 1996-97. Trop Med Int Health 1998;3:610-8 